\title{
On-body and Off-body Transmit Power Control in IEEE 802.15.6 Scheduled Access Networks
}

\author{
Fabio Di Franco, ${ }^{* \dagger} \mathrm{Yu} \mathrm{Ge}{ }^{\dagger}$ and Ilenia Tinnirello* \\ *Universita Degli Studi di Palermo, Italy \\ ${ }^{\dagger}$ Institute for Infocomm Research, Singapore \\ email: \{fabio.difranco, ilenia.tinnirello\}@tti.unipa.it, geyu@i2r.a-star.edu.sg
}

\begin{abstract}
Wireless Body Area Networks (WBANs) have received much attention due to the possibility to be used in healthcare applications. For these applications, energy saving is a critical issue, as in many cases, batteries cannot be easily replaced. A transmit power control scheme, able to adapt to the variations of the wireless body channel, will allow consistent energy saving and longer battery life.

In this paper we propose a transmit power control scheme suitable for IEEE 802.15.6 narrowband scheduled access networks, in which the transmission power is modulated frame by frame according to a run-time estimation of the channel propagation conditions. A simple and effective line search algorithm is proposed to estimate the channel quality based on the signal power received from the hub; in addition, an adaptive fade margin estimator is presented to determine an optimum margin based on the channel conditions. The approach allows tracking the highly variable propagation conditions due to the body mobility and the deployment of the sensors close to the human body. An experimental study in different test cases proves the effectiveness of the scheme in comparison with alternative solutions in the literature.
\end{abstract}

Index Terms-Wireless Body Area Network, Adaptive Transmit Power Control, IEEE 802.15.6, WBAN, ATPC

\section{INTRODUCTION}

Wireless Body Area Network (WBAN) devices have limited energy resources and are expected to operate over long periods of time without replacing batteries. A common approach to maximise battery lifetime is the use of power control algorithms which adjust the transmission power while ensuring successful delivery of frames. If the transmission power is selected too low, frames will be lost and retransmission costs counteract energy savings.

In this paper, we present an Adaptive Transmit Power Control (ATPC) scheme for WBAN links inferenced via RF signal strength and packet drop measurements at the receiver. It is suitable to work in IEEE 802.15.6 networks [1] without any modification to the standard.

The contribution of this paper is two-fold: firstly, when the IEEE 802.15.6 WBAN operates in beacon mode with superframe structure, the proposed ATPC scheme uses the beacon signal strength received by each sensor to predict channel gain in the subsequent scheduled access using a dynamic memory filter. Secondly, an opportunistic margin based on the channel gain stability is calculated to take into consideration channel gain variations due to fading and body movements.
Using the received signal strength indication (RSSI) to determine the transmit output power is a well established approach in wireless systems; the scheme presented in this paper differs because:

- it does not rely on information of sensor locations or control packets;

- it is able to adapt rapidly to abrupt changes in link conditions without overreacting

- it shows superior performance in terms of energy consumption but with low computation and memory requirements;

- it is suitable for IEEE 802.15.6 scheduled access MAC.

The scheme was tested in on-body to on-body and off-body to on-body scenario and its superior performance in terms of energy efficiency were experimentally proved for different activities and node locations.

The remainder of this manuscript is organised as follows. Section II presents the related work. Section III presents a short overview of the IEEE 802.15.6 standard and the scheduled allocation. Section IV describes the behaviour of the WBAN channels and introduces the ATPC algorithms. Section V compares the performance of our proposed ATPC with respect to alternative power tuning schemes.

\section{RELATED WORK}

Energy efficiency is one of the key requirements in WBAN. Jeong et al. [2] present a dynamic transmission-powercontrol algorithm in multi-hop Wireless Sensor Network based on the knowledge of signal strength received from the neighbours. Pavon et Choi [3] propose to monitor the Received Signal Strength Indicator (RSSI) from beacons or other frames sent by the access point and calculate the RSSI average using an exponential moving average filter. However, all these works are based on either theoretical analysis or simulations with idealized radio model, whereas we think that experimental studies in WBAN gives more realistic and meaningful results. [4][5] adapt the transmit power based on experimental results, but their investigations are done in wireless sensor networks, an environment significantly different from the WBAN.

More relevant to our work are, instead, the studies on adaptive power control protocol for WBAN based on experimental results: Moulton et al. [6] introduce a class of adaptive power control protocols, where the period between each feedback is varied according to the channel quality. Unfortunately, the 
needs of continuous control packets for sending feedback have a significant impact on energy consumption, as the authors have commented. Quwaider et al. [7] also present a closedloop power control algorithm for on-body wireless links based on the postural positions. Their algorithm promises to minimize the control overhead using a dynamic postural position inference. However, [8] shows that involuntary movements is the norm in human subject, making it hard to identify when a postural position is reached. Xiao et al. [9] present a Transmit Power Control scheme in an off-body to on-body environment. They propose a reactive scheme based on the exponential moving average of previous RSSIs. Our work is different from [9], as we propose an adaptive mechanism to dynamically tune the filter memory and the opportunistic margin based on the stability of previous RSSIs. We also investigate a new scenario (on-body to on-body) and add new activities/on-body positions. We compare the performances of both schemes using the IEEE 802.15.6 scheduled access mode and show the comparative performance in Section V.

\section{IEEE 802.15.6 SCHEDULED ALLOCATION}

The IEEE 802.15.6 working group has designed a standard for short-range wireless communications between a hub and devices in the vicinity of, or inside, a human body. The specification defines Narrowband (NB), Ultra-Wide Band (UWB) and Human Body Communications (HBC) physical layers. Each physical layer uses the existing industrial, scientific and medical (ISM) bands as well as the frequency bands approved by national medical and regulatory authority. A common MAC layer has been designed to be sufficiently flexible to allow different types of traffic and energy saving policies. The MAC can work either in beacon mode or non-beacon mode. In beacon mode, the hub of the star topology is responsible of managing the channel time in regular periods (called superframes) delimited by beacons. The superframe starts with a beacon frame followed by two consecutive periods, each consisting of an Exclusive Access Phase (EAP), a Random Access Phase (RAP), a Managed Access Phase (MAP), followed by an optional B2 frame and a Contention Access Phase (CAP). Our research focuses on the MAP which allows devices to access the channel in a scheduled (contention-free) manner. In the set up phase of a scheduled allocation, the devices send an allocation request to the hub, which specifies the data transfer direction, the wake-up period and the number of allocation slots required for transmission. The hub assigns the slots accordingly through an allocation response. Then the node can send data to the hub in the assigned interval.

\section{Transmit Power Control Scheme}

The WBAN links experience temporal variation of the signal conditions as the human moves and performs different activities. Figure 1 shows that RSSI variation reaches up to $20 \mathrm{~dB}$ when the orientation of the person changes and more than $30 \mathrm{~dB}$ during dynamic activities. The above results imply that an effective real-time transmit power control scheme, which adjusts the power based on the link conditions, is

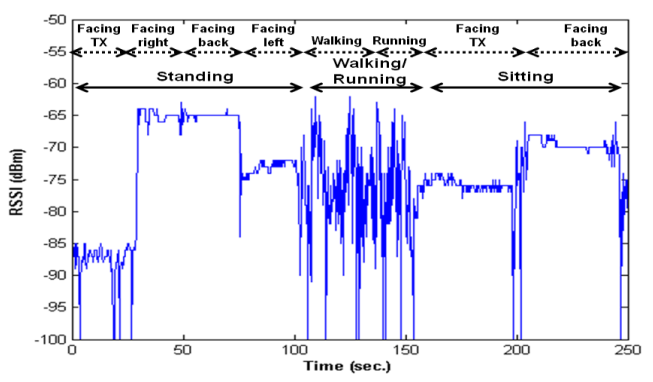

Fig. 1. RSSI Temporal variation in the off-body to on-body scenario with sensor receiver placed on arm (1)

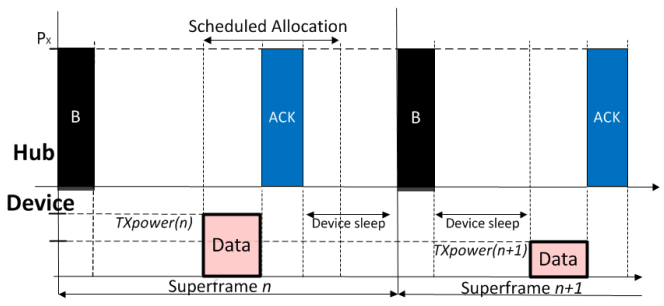

Fig. 2. Communication pattern between hub and each device

necessary to save energy and reduce external interference without compromising connection reliability.

\section{A. ATPC design}

The proposed ATPC for IEEE 802.15.6 scheduled access networks consists of three steps:

1) the adaptive channel gain predictor $\widehat{C}(n)$ is computed based on the historical RSSIs received from the devices, as explained in IV-B;

2) the fade margin estimator $\mu(n)$ is determined based on the channel stability, as shown in IV-C;

3 ) the instantaneous transmit output power $T X_{\text {Power }}(n)$ is calculated as

$$
T X_{\text {Power }}(n)=\widehat{C}(n)+\mu(n)-\text { sensitivity }
$$

where sensitivity is the nominal sensitivity value specified by the radio used.

We assume that the communications between the hub and each device happen during an up-link scheduled access communication, as described in Figure 2. At the beginning of the superframe $n$, the hub transmits beacon frames at maximum power $P_{X}$. Then each device transmits the data frame in the assigned scheduled allocation with transmit power $T X_{\text {Power }}(n)$ computed by the proposed ATPC. Following the successful reception of the data frame, the hub sends an acknowledgement at maximum power $P_{X}$, considering that the hub is not energy constrained as the devices.

\section{B. Channel Gain Predictor}

The prediction of the channel gain is the critical information in our ATPC scheme to determine the output power to be used in the subsequent frame. The channel gain is calculated at the 
beginning of each superframe as $R S S I_{\text {Beacon }}-P_{X}$, where $R S S I_{\text {Beacon }}$ is the signal strength of the beacon received by each device. We assume that the channel is reciprocal, i.e. the channel gain is the same in both directions [10]. In this way, each device is able to predict the channel gain after the reception of $n$-th beacon frame with the following autoregressive filter:

$$
\begin{aligned}
\widehat{C}(n)= & \alpha(n) \cdot\left(R S S I_{\text {Beacon }}(n)-P_{X}\right)+ \\
& +(1-\alpha(n)) \cdot \widehat{C}(n-1)
\end{aligned}
$$

where $\widehat{C}(n-1)$ is the previous channel gain estimate, and $\alpha(n)$ is the filter memory to be tuned according to the channel variability. In particular, for slow varying channels we can use larger $\alpha(n)$, giving more weight to the current channel sample, while for fast varying channels we can use smaller $\alpha(n)$, giving more weight to the channel history. In the former case, we will be able to track the actual channel dynamics, while in the latter case, we will track the slow dynamics only.

Upon the reception of the ACK, the mean squared error $M S E(n)$ can be calculated by comparing the estimate with the experienced channel gain, as :

$$
\operatorname{MSE}(n)=E\left[(\widehat{C}(n)-C(n))^{2}\right]
$$

where $C(n)$ is the instantaneous channel gain of the data frames at the superframe $n$.

Moreover, a simple and effective line search algorithm is proposed in Algorithm 1 to automatically determine the optimum $\alpha$ and minimise the error. After a beacon is received, three estimated channel gains are computed based on $\alpha, \alpha_{+}$ and $\alpha_{-}$, where $\alpha_{+}$and $\alpha_{-}$are calculated respectively through increasing and decreasing the current $\alpha$ by a constant $\epsilon$. After the scheduled allocation interval, the three MSEs are updated and the $\alpha$, associated with the minimum MSE, will be used in the next iteration.

\section{Opportunistic Fade Margin}

A fade margin, which is able to adapt in real time to the propagation conditions (i.e. sensor locations and human activities) without using any additional probe packets, is a critical element to guarantee high transmission reliability and low overhead. In this study, we propose to use the error provided by the channel gain predictor as a parameter for identifying the fading margin.

As shown in Algorithm 2, if the error is greater than the latest margin $\mu(n-1)$ by the threshold $T H R_{L}$, the margin is increased. Otherwise, if the error is less than the margin $\mu(n-1)$ by $T H R_{H}$, the margin is decreased. A minimum margin is also found in order to guarantee a successful data reception for each device. The adaptive minimum margin $T H R_{\min }$ is computed in real time at each device to take into account the sensitivity variance among radio chips. Lin et al. [11] highlighted that the difference in RSSIs between good and poor reception (i.e. low and high packet loss) is only a few $\mathrm{dB}$ in a low noise and low fading environment. This observation is applied in our design to determine the minimum



margin to add to the sensitivity level. In a stable wireless channel (i.e. $\sqrt{\widehat{M S E}(n)}<\delta$ )), our algorithm increases the $T H R_{\min }$ if a frame is lost; otherwise, the $T H R_{\min }$ is decreased if frames are consistently received successfully.

\section{EXPERIMENTAL EVALUATION}

This section presents the configurations of experiments and the performance evaluation of our ATPC scheme through both a temporal and a comparative statistical analysis.

\section{A. Setup and Scenarios}

The proposed adaptive transmit power control was tested in an IEEE 802.15.6 scheduled access network with a star topology, composed of a single hub node and a given number of body devices. 




Two scenarios were taken into consideration: the off-body to on-body scenario, where the hub node was placed on a table; the on-body to on-body scenario, where the hub was deployed on the right side of the waist. The seven nodes were placed on: right arm (1), left wrist (2), left side of the waist (3), right ankle (4), low centred back (5), high centred back (6) and head (7).

The experiments were conducted in a $9 \mathrm{~m} \times 9 \mathrm{~m}$ room in an office environment where a 24-year old male, with the height of $172 \mathrm{~cm}$ and a weight of $65 \mathrm{~kg}$, performed typical stationary and dynamic day-to-day activities.

In the off-body to on-body scenario, the subject was instructed to conduct the following activities: (a) stand for $100 \mathrm{~s}$ at $1.5 \mathrm{~m}$ away from the hub node, (b) sit for $100 \mathrm{~s}$ at $3 \mathrm{~m}$ away from the hub, (c) walk for $30 \mathrm{~s}$ and (d) run for $20 \mathrm{~s}$ on the walking path. In the on-body to on-body measurements the subject was instructed to conduct the following activities: (a) stand for $25 \mathrm{~s}$, (b) sit for $25 \mathrm{~s}$, (c) walk for the $30 \mathrm{~s}$ and (d) run for $20 \mathrm{~s}$. The sequence was repeated three times for validation purposes. During the experiments, a total of $170 \mathrm{k}$ samples were collected from the seven sensor nodes.

The experiments were conducted using a $250 \mathrm{~ms}$ long superframe. The algorithm and the code used for the validating and comparing the ATPCs is publicly released in [12].

\section{B. Experimental Results}

The proposed ATPC for IEEE 802.15.6 scheduled access networks consists of two components: the adaptive channel gain prediction (Algorithm 1) and the fade margin estimation
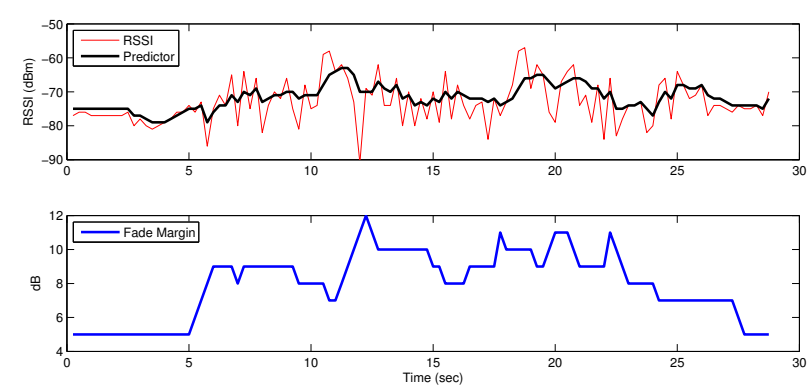

Fig. 3. Temporal snapshot of RSSI's vs. Channel Predictor and Fade margin.

(Algorithm 2). Figure 3 highlights the responsiveness of ATPC scheme at the abrupt variations of the propagation condition: the channel gain predictor follows the RSSI variation by adapting the filter memory accordingly and, when the variation are too fast for the predictor, the fade margin increases to prevent packet loss.

The performance of the proposed ATPC is also evaluated using two metrics: the power consumption for successfully received packet and the packet error rate (PER). The former is computed by dividing the total power consumed for all transmissions by the number of successfully delivered packets. The power consumption is the one drawn by the CC 2420 transceiver [13] used in our experiments. The proposed scheme does not introduce any control message such that the power consumption only includes the cost of data transmissions.

The performance evaluation is conducted through comparing our proposed ATPC with alternative power tuning solutions as the one proposed in [9] and the fixed TX output power level equal to $0 \mathrm{dBm},-10 \mathrm{dBm}$ and $-15 \mathrm{dBm}$.

Figure 4 shows the performance when devices are deployed at different positions in the off-body to on-body scenario. For all the positions, on average, our proposed scheme achieves a power saving of $11 \%$ with respect to [9], 39\% with respect to the fixed $0 \mathrm{dBm}$ output power. In this scenario the PER is less than 3\% for all the positions, both in [9] and our algorithm.

Figure 5a shows the power consumptions in the on-body to on-body scenario. In this case, on average, our proposed ATPC saves $17 \%$ with respect to [9], 33\% with respect to the fixed $0 \mathrm{dBm}$ and $14 \%$ with respect to the fixed $-10 \mathrm{dBm}$. However the link between the waist and the high back suffers a PER of $5 \%$ at $0 \mathrm{dBm}$ and $35 \%$ at $-10 \mathrm{dBm}$. It indicates that a relay mechanism will be beneficial in such cases.

Figure $5 \mathrm{~b}$ shows that the power consumptions across different activities. The power consumption at fixed TX power levels is driven higher in the dynamic activities due to the higher packet loss. Surprisingly, in the on-body scenario with the sit posture, the power consumption is higher than other activities. This is due to the RF degradation caused by the close proximity of the hub antenna, located on the waist, to the hip, when sitting. Averaging the measured power consumption across all the activities and positions, our scheme saves $16 \%$ with respect to [9] and 37\% with respect to a fixed TX power of $0 \mathrm{dBm}$, while limiting the average PER to less than $3 \%$. 


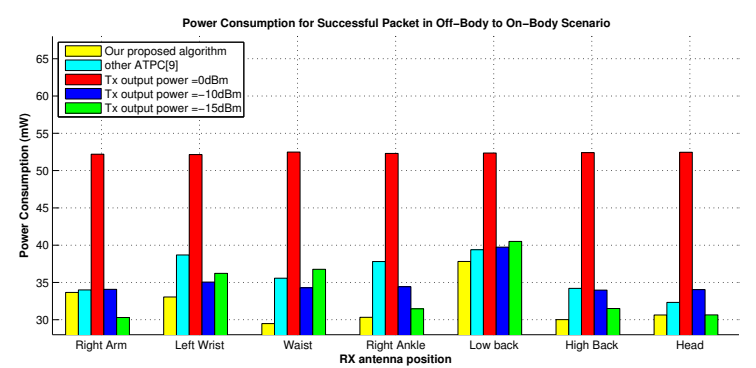

(a)

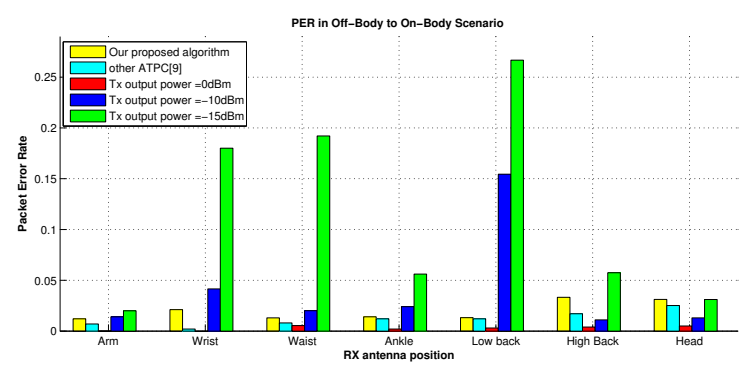

(b)

Fig. 4. Power consumption (a) and PER (b) for different ATPC scheme in off-body to on-body scenario.

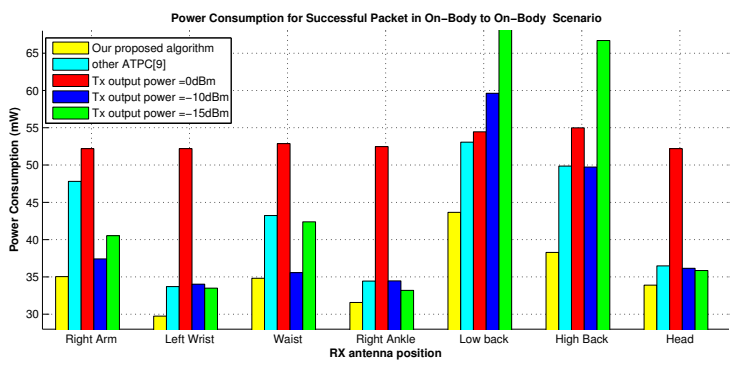

(a)

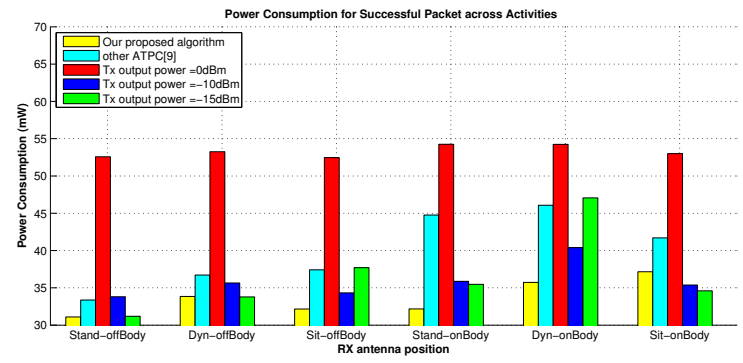

(b)

Fig. 5. Power consumption for different ATPC scheme in on-body to on-body scenario (a) and across different activities (b).

\section{CONCLUSion AND Future Work}

This paper presented an adaptive transmit power control scheme to ensure reliable and energy efficient transmissions in time-varying body channels. The proposed algorithm is suitable to be used in the IEEE 802.15.6 beacon mode MAC with superframe structure. It does not introduce any probe packet and does not require any modification of the standard for interoperability.

The proposed ATPC was compared with fixed output power transmissions and another WBAN ATPC algorithm [9]. The comparison was performed on WBAN scenarios with a sequence of daily activities.

The measurement results show that the proposed ATPC achieves better energy efficiency without compromising transmission reliability: the energy saved is on average $16 \%$ across all activities with respect to [9] and $37 \%$ with respect to a fixed $0 \mathrm{dBm}$ TX power, while achieving an average PER less than $3 \%$. It motivates us to design an effective relay mechanism to reduce the PER and the global power consumption, which will be our future research.

\section{REFERENCES}

[1] "IEEE Standard for Local and metropolitan area networks Part 15.6: Wireless Body Area Networks," IEEE Std 802.15.6-2012, pp. 1 -271, 29 Feb. 2012.

[2] J. Jeong, D. Culler, and J.-H. Oh, "Empirical analysis of transmission power control algorithms for wireless sensor networks," in International Conference on INSS 2007, June 2007, pp. 27 -34.

[3] J. Pavon and S. Choi, "Link adaptation strategy for ieee 802.11 wlan via received signal strength measurement," in International Conference on ICC 2003, vol. 2, May 2003, pp. 1108 -1113 vol.2.
[4] D. Son, B. Krishnamachari, and J. Heidemann, "Experimental study of the effects of transmission power control and blacklisting in wireless sensor networks," in IEEE SECON 2004., Oct. 2004, pp. 289 - 298.

[5] M. Raza, G. Ahmed, and N. M. Khan, "Experimental evaluation of transmission power control strategies in wireless sensor networks," in International Conference on Emerging Technologies, Oct. 2012, pp. 1 -4 .

[6] B. Moulton, L. Hanlen, J. Chen, G. Croucher, L. Mahendran, and A. Varis, "Body-area-network transmission power control using variable adaptive feedback periodicity," in Australian Communications Theory Workshop (AusCTW), Feb. 2010, pp. 139 -144.

[7] M. Quwaider, J. Rao, and S. Biswas, "Transmission power assignment with postural position inference for on-body wireless communication links," ACM Trans. Embed. Comput. Syst., vol. 10, no. 1, pp. 14:114:27, Aug. 2010.

[8] F. Di Franco, C. Tachtatzis, B. Graham, M. Bykowski, D. Tracey, N. Timmons, and J. Morrison, "The effect of body shape and gender on wireless body area network on-body channels," in IEEE Middle East Conference on Antennas and Propagation (MECAP), Oct. 2010, pp. 1 -3 .

[9] S. Xiao, A. Dhamdhere, V. Sivaraman, and A. Burdett, "Transmission power control in body area sensor networks for healthcare monitoring," IEEE Journal on Selected Areas in Communications, vol. 27, no. 1, pp. $37-48$, January 2009.

[10] L. Hanlen, V. Chaganti, B. Gilbert, D. Rodda, T. Lamahewa, and D. Smith, "Open-source testbed for body area networks: 200 sample/sec, 12 hrs continuous measurement," in PIMRC 2010, Sept. 2010, pp. 66 -71 .

[11] S. Lin, J. Zhang, G. Zhou, L. Gu, J. A. Stankovic, and T. He, "Atpc: adaptive transmission power control for wireless sensor networks," in Proceedings of ACM SenSys 2006, 2006, pp. 223-236.

[12] "TinyOS code for validating and comparing ATPC algorithms." [Online]. Available: http://code.google.com/p/rewin

[13] CC2420 Single-Chip 2.4 GHz IEEE 802.15.4 Compliant and ZigBee Ready RF Transceiver. [Online]. Available: http://www.ti.com/lit/ds/symlink/cc2420.pdf 